Bull. Soc. math. France

130 (4), 2002, p. 619-626

\title{
COINCIDENCE FOR SUBSTITUTIONS OF PISOT TYPE
}

\author{
By Marcy Barge \& Beverly Diamond
}

\begin{abstract}
Let $\varphi$ be a substitution of Pisot type on the alphabet $\mathcal{A}=\{1,2, \ldots, d\}$; $\varphi$ satisfies the strong coincidence condition if for every $i, j \in \mathcal{A}$, there are integers $k, n$ such that $\varphi^{n}(i)$ and $\varphi^{n}(j)$ have the same $k$-th letter, and the prefixes of length $k-1$ of $\varphi^{n}(i)$ and $\varphi^{n}(j)$ have the same image under the abelianization map. We prove that the strong coincidence condition is satisfied if $d=2$ and provide a partial result for $d \geq 2$.

RÉSUMÉ (Coïncidence pour les substitutions de type Pisot). - Soit $\varphi$ une substitution de type Pisot sur un alphabet $\mathcal{A}=\{1,2, \ldots, d\}$; on dit que $\varphi$ satisfait la condition de coïncidence forte si pour tout $i, j \in \mathcal{A}$, il existe des entiers $k, n$ tels que $\varphi^{n}(i)$ et $\varphi^{n}(j)$ aient la même $k$-ième lettre et les préfixes de longueur $k-1$ de $\varphi^{n}(i)$ et $\varphi^{n}(j)$ aient la même image par l'application d'abélianisation. Nous montrons que la condition de coïncidence forte est satisfaite pour $d=2$ et nous donnons un résultat partiel pour $d \geq 2$.
\end{abstract}

A substitution $\varphi$ on a finite alphabet $\mathcal{A}=\{1,2, \ldots, d\}$ satisfies the strong coincidence condition if for every $i, j \in \mathcal{A}$, there are integers $k, n$ such that

(i) $\varphi^{n}(i)$ and $\varphi^{n}(j)$ have the same $k$-th letter and

Texte reçu le 21 novembre 2001, accepté le 14 mai 2002

Marcy BArge, Department of Mathematics, Montana State University, Bozeman MT

59717 (USA) • E-mail : barge@math.montana.edu

Beverly Diamond, Department of Mathematics, College of Charleston, Charleston SC 29424

(USA) - E-mail : diamondb@cofc.edu

2000 Mathematics Subject Classification. - 37B10.

Key words and phrases. - Substitution, dynamical system, Pisot, coincidence conjecture, pure discrete spectrum.

The second author was partially supported by a research incentive grant from the South Carolina Commission on Higher Education.

BULletin DE LA SOCiÉTÉ MATHÉMATIQUE DE FRANCE (C) Société Mathématique de France

$0037-9484 / 2002 / 619 / \$ 5.00$ 
(ii) the prefixes of length $k-1$ of $\varphi^{n}(i)$ and $\varphi^{n}(j)$ have the same image under the abelianization map (that is, these prefixes contain the same number of occurrences of each $i \in \mathcal{A}$ ).

This condition (without the requirement on prefixes) was first introduced for substitutions of constant length by F.M. Dekking [5], who proved that for such substitutions, the condition is satisfied if and only if the associated substitutive dynamical system has pure discrete spectrum (equivalently, the substitutive system is metrically isomorphic with translation on a compact abelian group). In its current form, the strong coincidence condition is due to Arnoux and Ito. The equivalence of the strong coincidence condition and pure discrete spectrum also holds for substitutions of nonconstant length of Pisot type on two letters (unpublished work of B. Host, [8], [9], [6], and [7]). According to [4], Host also proved that if $\varphi$ is a Pisot type substitution on two letters which is unimodular (its transition matrix has determinant +1 or -1 ) and which satisfies the strong coincidence condition, then the substitutive system associated with $\varphi$ is metrically isomorphic with both an interval exchange and a one-dimensional toral rotation. Arnoux and Ito [1] have recently shown that every unimodular Pisot substitution on $d \geq 2$ letters satisfying the strong coincidence condition is metrically isomorphic with an exchange of domains in $\mathbb{R}^{d-1}$ and is semi-conjugate to a rotation on the $d$-1-torus (see also [11]). At present, an additional condition is needed to improve the semi-conjugacy to metric isomorphism. For examples of nonunimodular Pisot substitutive systems that are metrically isomorphic with translations on $p$-adic groups and $p$-adic solenoids, see $[10]$.

No examples of Pisot type substitutions which do not satisfy the strong coincidence condition are known, and the statement that all such substitutions satisfy this condition is known as the Strong Coincidence Conjecture. In this paper, we prove that the Strong Coincidence Conjecture holds for Pisot substitutions on two letters and provide a partial result for substitutions on $d$ letters where $d>2$.

Theorem 1. - Let $\varphi$ be a Pisot substitution on an alphabet $\mathcal{A}=\{1,2, \ldots, d\}$. There are distinct letters $i, j \in \mathcal{A}$ for which there are integers $k, n$ such that $\varphi^{n}(i)$ and $\varphi^{n}(j)$ have the same $k$-th letter, and the prefixes of length $k-1$ of $\varphi^{n}(i)$ and $\varphi^{n}(j)$ have the same image under the abelianization map.

We introduce terminology necessary for the proof of the theorem.

For $j=1$ to $d, I_{j}$ will denote the interval of length 1 in $\mathbb{R}^{d}$ given by

$$
I_{j}=\{0\} \times\{0\} \times \cdots \times[0,1] \times \cdots \times\{0\},
$$

where $[0,1]$ appears in the $j$-th position.

TOME $130-2002-\mathrm{N}^{\mathrm{O}} 4$ 
Similarly, for $1 \leq j \leq d, \boldsymbol{e}_{j}$ will denote the unit vector $(0,0, \ldots, 1, \ldots, 0)$, where 1 appears in the $j$-th position. If $x \in \mathbb{Z}^{d}$, the integer lattice in $\mathbb{R}^{d}$, the vector from the origin to $x$ is denoted by $\boldsymbol{x}$.

For $\boldsymbol{v}=\left(v_{1}, v_{2}, \ldots, v_{d}\right)$,

$$
I_{j}+\boldsymbol{v}=\left\{v_{1}\right\} \times\left\{v_{2}\right\} \times \cdots \times\left[v_{j}, v_{j}+1\right] \times \cdots \times\left\{v_{d}\right\} .
$$

The collection $\mathcal{M}$ of line segments joining 'adjacent' elements of the integer lattice is then

$$
\mathcal{M}=\left\{I_{j}+\boldsymbol{x}: 1 \leq j \leq d, x \in \mathbb{Z}^{d}\right\} .
$$

The term segment will refer to an element of $\mathcal{M}$.

Let $\mathcal{A}=\{1,2, \ldots, d\}$ be a finite alphabet and $\mathcal{A}^{*}$ the collection of finite non-empty words formed from the alphabet $\mathcal{A}$.

A substitution $\varphi$ is a map $\varphi: \mathcal{A} \rightarrow \mathcal{A}^{*} ; \varphi$ has an associated transition matrix $A_{\varphi}=A=\left(a_{i j}\right)_{i, j \in \mathcal{A}}$ in which $a_{i j}$ is the number of occurrences of $i$ in the word $\varphi(j)$. (In what follows, we will also use $A$ to denote the linear transformation on vectors of $\mathbb{R}^{d}$ and the linear function acting on points of $\mathbb{R}^{d}$.)

The substitution $\varphi$ is primitive if there is $n$ so that for each $i, j \in \mathcal{A}, j$ appears in $\varphi^{n}(i) ; \varphi$ is of Pisot type, or simply Pisot, if all eigenvalues of $A$ other than the Perron-Frobenius eigenvalue have modulus strictly between 0 and 1 .

If $\varphi$ is Pisot, then $\varphi$ is primitive and the (hyperbolic) linear map on $\mathbb{R}^{d}$ defined by the matrix $A$ has stable space $E^{s}$ of dimension $d-1$ and unstable space $E^{u}$ of dimension 1 spanned by a positive Perron-Frobenius eigenvector $\boldsymbol{v}_{u}$. Also, neither $E^{s}$ nor $E^{u}$ contain elements of the integer lattice other than the origin. (See Chapter 1 of [3], for instance.)

Assume for the remainder of this paper that $\varphi$ is Pisot.

We define an order on $\mathbb{Z}^{d}$ as follows. Given $P_{1}, P_{2} \in \mathbb{Z}^{d}, P_{1} \leq P_{2}$ if there are $t_{1} \leq t_{2} \in \mathbb{R}$ so that $P_{1} \in E^{s}+t_{1} \boldsymbol{v}_{u}, P_{2} \in E^{s}+t_{2} \boldsymbol{v}_{u}$ (we use $E^{s}+t \boldsymbol{v}_{u}$ to denote the set of points $x$ such that $\boldsymbol{x}=\boldsymbol{w}+t \boldsymbol{v}_{u}$ for some $\left.\boldsymbol{w} \in E^{s}\right)$. Note that if $P_{1}, P_{2} \in \mathbb{Z}^{d}$, and $P_{1} \leq P_{2}$, then $A P_{1} \leq A P_{2}$.

We employ a geometrical formulation of substitution developed by Arnoux, Ito and Sano [2]. The inflation and substitution map $F_{\varphi}$ is defined on subsets of the set $\mathcal{M}$ of line segments in the following manner. Suppose that

$$
\varphi(j)=a(j, 1) a(j, 2) \cdots a(j, n(j)), \quad \text { for } \quad 1 \leq j \leq d .
$$

Define

$$
\begin{aligned}
\boldsymbol{v}_{\varphi}(j, 0) & =\mathbf{0} \\
\boldsymbol{v}_{\varphi}(j, i) & =\boldsymbol{v}_{\varphi}(j, i-1)+\boldsymbol{e}_{a(j, i)}, \quad 1 \leq i \leq n(j) .
\end{aligned}
$$

Then

$$
\begin{aligned}
F_{\varphi}\left(I_{j}\right) & =\left\{I_{a(j, i)}+\boldsymbol{v}_{\varphi}(j, i-1): 1 \leq i \leq n(j)\right\}, \\
F_{\varphi}\left(I_{j}+\boldsymbol{v}\right) & =F_{\varphi}\left(I_{j}\right)+A \boldsymbol{v}=\left\{s+A \boldsymbol{v}: s \in F_{\varphi}\left(I_{j}\right)\right\} .
\end{aligned}
$$

BULletin DE LA SOCiÉtÉ MATHÉMATIQUE DE FRANCE 
Finally, if $M \subseteq \mathcal{M}$,

$$
F_{\varphi}(M)=\{F(s): s \in M\}
$$

(Strictly speaking, in the above, we should write $F_{\varphi}\left(\left\{I_{j}\right\}\right)$ rather than $F_{\varphi}\left(I_{j}\right)$, etc., but we abuse notation and use the latter when convenient.) Note that

$$
F_{\varphi^{n}}=F_{\varphi}^{n} \text {. }
$$

Suppose that

$$
S=\left\{s_{i}=I_{s(i)}+\boldsymbol{v}_{i}: 1 \leq i \leq k, 1 \leq s(i) \leq d\right\}
$$

is a non-empty finite collection of segments, i.e., a subset of $\mathcal{M}$. The set $S$ is a strand if for each $1 \leq i \leq k-1$,

$$
\boldsymbol{v}_{i+1}=\boldsymbol{v}_{i}+\boldsymbol{e}_{s(i)}
$$

The word associated with $S$ is $w_{S}=s(1) s(2) \cdots s(k)$. For such a word $w$,

$$
\boldsymbol{l}(w)=\left(w_{1}, w_{2}, \ldots, w_{d}\right)
$$

where for each $i \in \mathcal{A}, w_{i}=\#$ of occurrences of $i$ in $W$. The displacement vector for $S$ is then $\boldsymbol{l}(S)=\boldsymbol{l}\left(w_{S}\right)$ and the length of $S$ is $|S|=|\boldsymbol{l}(S)|$, where

$$
\left|\left(v_{1}, v_{2}, \ldots, v_{d}\right)\right|=\sum_{1 \leq j \leq d}\left|v_{j}\right| .
$$

The endpoints of the segments belonging to $S$ will be called vertices of $S$. A strand $S$ has an initial vertex $P$ and a terminal vertex $Q$ with the property that $P \leq R \leq Q$ for all vertices $R$ of $S$; we say that $S$ is a strand from $P$ to $Q$. The segments containing $P$ and $Q$ are the initial and terminal segments of $S$, respectively. Note that if $S$ is a strand from $P$ to $Q$, then $F_{\varphi}(S)$ is a strand from $A P$ to $A Q$. Two strands $S_{1}$ and $S_{2}$ are coincident if $S_{1} \cap S_{2} \neq \varnothing$ (that is, they share a segment), and eventually coincident if $F_{\varphi}^{m}\left(S_{1}\right) \cap F_{\varphi}^{m}\left(S_{2}\right) \neq \varnothing$ for some $m \in \mathbb{N}$.

The coincidence conjecture can then be stated as follows:

Coincidence Conjecture. - For $1 \leq j, r \leq d, I_{j}$ and $I_{r}$ are eventually coincident.

We prove the following:

THEOREM 1. - There are $1 \leq j \neq r \leq d$ so that $I_{j}$ and $I_{r}$ are eventually coincident.

In the following, $N_{B}\left(E^{u}\right)$ will denote $\left\{x \in \mathbb{R}^{d}: \operatorname{dist}\left(x, E^{u}\right)<B\right\}$ where $\operatorname{dist}(x, y)=|\boldsymbol{x}-\boldsymbol{y}|$, the usual Euclidean distance between $x$ and $y$.

LemmA 2. - There are $B>0$ and $n \in \mathbb{N}$ so that

(i) if a segment $s$ lies in $N_{B}\left(E^{u}\right)$, then the strand $F_{\varphi}^{n}(s)$ lies in $N_{B}\left(E^{u}\right)$ and

(ii) for any segment $s$, there is $k \in \mathbb{N}$ so that $F_{\varphi}^{n k}(s)$ lies in $N_{B}\left(E^{u}\right)$.

TOME $130-2002-\mathrm{N}^{\mathrm{O}} 4$ 
Proof. - The fact that $\varphi$ is Pisot implies that there are $\lambda, 0<\lambda<1$, and a constant $C$ so that $\left|A^{n} \boldsymbol{v}\right|<C \lambda^{n}|\boldsymbol{v}|$ for all $\boldsymbol{v} \in E^{s}$. It follows that there are $n$ and $\lambda^{\prime}$ with $\lambda<\lambda^{\prime}<1$ so that $A^{n}\left(N_{B}\left(E^{u}\right)\right) \subseteq N_{\lambda^{\prime} B}\left(E^{u}\right)$ for all $B>0$.

Now suppose that $s$ is any segment lying in $N_{B}\left(E^{u}\right)$. The initial point of $F_{\varphi}^{n}(s)$ lies in $N_{\lambda^{\prime} B}\left(E^{u}\right)$ and the entire strand $F_{\varphi}^{n}(s)$ lies in $N_{\lambda^{\prime} B+m}\left(E^{u}\right)$, where $m$ is the maximum of the lengths of $\varphi^{n}(i)$ for $i \in \mathcal{A}$. Choose $B$ large enough so that $\lambda^{\prime} B+m<\lambda^{\prime \prime} B$ for some $\lambda^{\prime \prime}, 0<\lambda^{\prime \prime}<1$. For such $B$ and any segment $s$ in $N_{B}\left(E^{u}\right), F_{\varphi}^{n}(s)$ lies in $N_{B}\left(E^{u}\right)$.

If $s$ is a segment not in $N_{B}\left(E^{u}\right)$, let $B^{\prime}$ be large enough so that $s$ lies in $N_{B^{\prime}}\left(E^{u}\right)$. If $k \geq 1$ is such that $\left(\lambda^{\prime \prime}\right)^{k} B^{\prime} \leq B$, then $F_{\varphi}^{k n}(s)$ lies in $N_{B}\left(E^{u}\right)$.

Since $F_{\varphi^{n}}=F_{\varphi}^{n}, I_{j}$ and $I_{r}$ are eventually coincident under $F_{\varphi}$ if and only if they are eventually coincident under $F_{\varphi^{n}}$. Thus we assume for the remainder of this paper that the $n$ of Lemma 2 is $n=1$.

A configuration of segments is a collection $\mathcal{C}$ of segments with the property that $E^{s}+\boldsymbol{v}$ intersects each element of $\mathcal{C}$ in an interior point for some $\boldsymbol{v}$. A configuration of strands is a collection $\mathcal{C}$ of strands with the property that both the collections of initial segments and final segments form configurations of segments. The size of a configuration $\mathcal{C}$ of strands is the number of strands in $\mathcal{C}$. If $P^{\prime}$ is the largest of the initial vertices of strands of $\mathcal{C}$ and $Q^{\prime}$ is the smallest of the terminal vertices of strands of $\mathcal{C}$, we say that $\mathcal{C}$ is a configuration of strands from $P^{\prime}$ to $Q^{\prime}$, or that $\mathcal{C}$ extends from $P^{\prime}$ to $Q^{\prime}$, with length equal to $\left|\boldsymbol{Q}^{\prime}-\boldsymbol{P}^{\prime}\right|$.

Given a configuration $\mathcal{C}$ of strands from $P^{\prime}$ to $Q^{\prime}$, there is a unique configuration of strands $\mathcal{C}^{(1)} \subseteq F_{\varphi}(\mathcal{C})$ with the following properties:

(i) $\mathcal{C}^{(1)}$ has the same size as $\mathcal{C}$;

(ii) $\mathcal{C}^{(1)}$ extends from $A P^{\prime}$ to $A Q^{\prime}$; and

(iii) for each strand $S^{\prime} \in \mathcal{C}^{(1)}$, there is a strand $S \in \mathcal{C}$ with $S^{\prime} \subseteq F_{\varphi}(S)$.

We call $\mathcal{C}^{(1)}$ the first iterate of $\mathcal{C}$; higher iterates are defined by

$$
\mathcal{C}^{(2)}=\left(\mathcal{C}^{(1)}\right)^{(1)}, \text { etc. }
$$

A configuration $\mathcal{C}$ of strands is not eventually coincident if each distinct pair of strands of $\mathcal{C}$ is not eventually coincident.

The following is a consequence of the definitions and Lemma 2.

Lemma 3. - Suppose that $\mathcal{C}$ is a not eventually coincident configuration of strands of size $m$. The iterates $\mathcal{C}^{(k)}$ of $\mathcal{C}$ satisfy the following:

(i) $\mathcal{C}^{(k)}$ is a not eventually coincident configuration of strands;

(ii) length $\left(\mathcal{C}^{(k)}\right) \rightarrow \infty$ as $k \rightarrow \infty$; and

(iii) there is a positive integer $K$ so that $\mathcal{C}^{(k)}$ lies in $N_{B}\left(E^{u}\right)$ for all $k \geq K$.

LEMmA 4. - There is an integer $M$ with the following properties: 
(i) there exists a configuration of segments of size $M$ that is not eventually coincident; and

(ii) every configuration of segments of size larger than $M$ is eventually coincident.

Proof. - For each $t \in \mathbb{R}$, let $n(t)$ be the number of distinct segments lying in $N_{B}\left(E^{u}\right)$ that meet $E^{s}+t \boldsymbol{v}_{u}$ and let $N=\sup _{t \in \mathbb{R}} n(t)$. Then $N<\infty$. Let $\mathcal{C}$ be a configuration of not eventually coincident segments of size $m$ and choose $k$ large enough so that $\mathcal{C}^{(k)}$ lies in $N_{B}\left(E^{u}\right)$. For some $t, E^{s}+t \boldsymbol{v}_{u}$ contains no vertices of $\mathcal{C}^{(k)}$ and meets a segment from each strand of $\mathcal{C}^{(k)}$. Since the strands of $\mathcal{C}^{(k)}$ are not coincident, $m \leq N$. Then $N$ is an upper bound on the size of not eventually coincident configurations.

Proof of Theorem 1. - Let $\mathcal{C}$ be a not eventually coincident configuration of segments of maximum size $M$ as in Lemma 4 . Without loss of generality, $\mathcal{C}^{(k)}$ lies in $N_{B}\left(E^{u}\right)$ for all $k \geq 0$. For each $k$, let $P_{0}<P_{1}<\cdots<P_{m}\left(P_{i}=P_{i}(k)\right)$ be the vertices of $\mathcal{C}^{(k)}$ that lie between the initial vertex $P_{0}$ and the terminal vertex $P_{m}$. Let $t_{0}<t_{1}<\cdots<t_{m}\left(t_{i}=t_{i}(k)\right)$ be such that $P_{i}$ lies on $E^{s}+t_{i} \boldsymbol{v}_{u}$. For $1 \leq i \leq m$, let

$$
\begin{array}{r}
\mathcal{C}_{i}^{(k)}=\left\{s: s \text { is a segment of some strand of } \mathcal{C}^{(k)}\right. \text { and } \\
\left.\qquad s \text { meets } E^{s}+t \boldsymbol{v}_{u} \text { for } t_{i-1}<t<t_{i}\right\} .
\end{array}
$$

Then $\mathcal{C}_{i}^{(k)}$ is a configuration of segments of size $M$ (one from each strand of $\mathcal{C}^{(k)}$ ) that is not eventually coincident and that extends from $P_{i-1}$ to $P_{i}$. Let $\ell_{i} \geq 1$ be the number of segments of $\mathcal{C}_{i}^{(k)}$ that terminate at $P_{i}$. Then exactly $\ell_{i}$ of the segments of $\mathcal{C}_{i+1}^{(k)}$ begin at $P_{i}$. Let $\mathcal{S}_{i+1}$ denote the collection of segments of $\mathcal{C}_{i+1}^{(k)}$ issuing from $P_{i}$, let $\mathcal{S}_{i+1}^{\prime}$ be any collection of $\ell_{i}$ (distinct) segments beginning at $P_{i}$ different from $\mathcal{S}_{i+1}$, if there is such a collection, and consider the collection

$$
\mathcal{C}_{i+1}^{\prime}=\left(\mathcal{C}_{i+1}^{(k)} \backslash \mathcal{S}_{i+1}\right) \cup \mathcal{S}_{i+1}^{\prime} .
$$

Suppose that for some $\mathcal{S}_{i+1}^{\prime}, \mathcal{C}_{i+1}^{\prime}$ is not eventually coincident. The maximality of $\mathcal{C}_{i+1}^{(k)}$ implies that $\mathcal{C}_{i+1}^{(k)} \cup \mathcal{S}_{i+1}^{\prime}$ is eventually coincident, hence there are

$$
s \in \mathcal{S}_{i+1}, \quad s^{\prime} \in \mathcal{S}_{i+1}^{\prime}, \quad s \neq s^{\prime},
$$

such that $\left\{s, s^{\prime}\right\}$ is eventually coincident. Write

$$
s=I_{j}+\boldsymbol{v}, \quad s^{\prime}=I_{r}+\boldsymbol{v} .
$$

It follows that $I_{j}$ and $I_{r}$ are eventually coincident, and the theorem is proved.

Suppose then, for the remainder of this proof, that for each $\mathcal{S}_{i+1}^{\prime} \neq \mathcal{S}_{i+1}$, $\mathcal{C}_{i+1}^{\prime}$ is eventually coincident. Then $\mathcal{C}_{i}^{(k)}$ completely determines $\mathcal{C}_{i+1}^{(k)}$.

TOME $130-2002-\mathrm{N}^{\mathrm{O}} 4$ 
In the above argument, the location of configurations did not play a role; only the relative position of segments within configurations was considered. In particular, if there are $p$ with $0 \leq i \leq i+p<m$ and $\boldsymbol{v}$ so that

$$
\mathcal{C}_{i+p}^{(k)}=\mathcal{C}_{i}^{(k)}+\boldsymbol{v}, \quad \text { then } \quad \mathcal{C}_{i+p+1}^{(k)}=\mathcal{C}_{i+1}^{(k)}+\boldsymbol{v} .
$$

Up to translation, there are only finitely many configurations of segments that lie in $N_{B}\left(E^{u}\right)$; let $b$ be an upper bound on the number of equivalence classes (up to translation) of such configurations of segments. Recall that $\mathcal{C}^{(k)}$ extends from $P_{0}$ to $P_{m}(m=m(k))$ and that $P_{0}<P_{1}<\cdots<P_{m}\left(P_{i}=P_{i}(k)\right)$ are the vertices of $\mathcal{C}^{(k)}$ that lie between $P_{0}$ and $P_{m}$. The vectors

$$
\boldsymbol{P}_{i+1}-\boldsymbol{P}_{i}, \quad 0 \leq i \leq m-1,
$$

are bounded independently of $m$, and length $\left(\mathcal{C}^{(k)}\right)=\left|\boldsymbol{P}_{m}-\boldsymbol{P}_{0}\right| \rightarrow \infty$ as $k \rightarrow$ $\infty$. Thus $m(k) \rightarrow \infty$ as $k \rightarrow \infty$ and, for $m(k)>b$, there are $i=i(k) \leq b$ and $\ell$ with $i<\ell \leq b \leq m(k)$ such that $\mathcal{C}_{\ell}^{(k)}=\mathcal{C}_{i}^{(k)}+\boldsymbol{v}$ for the integer vector $\boldsymbol{v}=\boldsymbol{P}_{\ell}-\boldsymbol{P}_{i}$. It follows from the above that

$$
\mathcal{C}_{i+p(\ell-i)}^{(k)}=\mathcal{C}_{i}^{(k)}+p \boldsymbol{v}
$$

for all positive integers $p$ with $i+p(\ell-i) \leq m$. Since $i<\ell \leq b$ and $\boldsymbol{v}$ is a nonzero integer vector bounded independently of $k$, some such vector $\boldsymbol{v}$ occurs for infinitely many $k$. For this $\boldsymbol{v}$, it must be the case that $p \boldsymbol{v}$ lies in $N_{2 B}\left(E^{u}\right)$ for arbitrarily large $p$. But then $\boldsymbol{v}$ is a scalar multiple of $\boldsymbol{v}_{u}$, so that $E^{u}$ contains a nonzero point of $\mathbb{Z}^{d}$, an impossibility. This contradiction proves the theorem.

By graphing the fixed words, the reader can verify easily that neither of the (non-Pisot) substitutions

$$
\begin{gathered}
\varphi(1)=121, \quad \varphi(2)=221, \\
\tau(1)=12, \quad \tau(2)=21,
\end{gathered}
$$

satisfies the Strong Coincidence Conjecture.

Acknowledgements. - We would like to thank P.Arnoux, A.Siegel and C. Holton for introducing us to the Strong Coincidence Conjecture and reading an early draft of this manuscript.

\section{BIBLIOGRAPHY}

[1] Arnoux (P.) \& Ito (S.) - Pisot substitutions and Rauzy fractals, Bull. Belg. Math. Soc. Simon Stevin, t. 8 (2001), pp. 181-207.

[2] Arnoux (P.), Ito (S.) \& SAno (Y.) - Higher dimensional extensions of substitutions and their dual maps, J. Anal. Math., t. 83 (2001), pp. 183206.

BULletin DE LA SOCiÉtÉ MATHÉmATiQUe DE FRANCE 
[3] Berthé (V.), Ferenczi (S.), Mauduit (C.) \& Siegel (A.), eds. Introduction to Finite Automata and Substitutions Dynamical Systems, Lectures Notes in Mathematics, Springer-Verlag, to appear, http://iml. univ-mrs.fr/editions/preprint00/book/prebookdac.html.

[4] Canterini (V.) \& Siegel (A.) - Geometric representation of primitive substitutions of Pisot type, Trans. Amer. Math. Soc., t. 353 (2001), pp. 5121-5144.

[5] DeKking (F.M.) - The spectrum of dynamical systems arising from substitutions of constant length, Z. Wahrscheinlichkeitstheorie und Verw. Gebiete, t. 41 (1977/78), pp. 221-239.

[6] Hollander (M.) - Linear numeration systems, finite $\beta$-expansions, and discrete spectrum of substitution dynamical systems, Ph.D. Thesis, University of Washington, 1996.

[7] Hollander (M.) \& Solomyak (B.) - Two-symbol Pisot substitutions have pure discrete spectrum, preprint to appear in Ergodic Theory and Dynamical Systems.

[8] Livshits (A.N.) - On the spectra of adic transformations of Markov compact sets, Uspekhi Mat. Nauk, t. 42 (1987), pp. 189-190, English translation: Russian Math. Surveys 42 (1987), p. 222-223.

[9] _ Sufficient conditions for weak mixing of substitutions and of stationary adic transformations, Mat. Zametki, t. 44 (1988), pp. 785-793, 862, English translation: Math. Notes 44 (1988), p. 920-925.

[10] Siegel (A.) - Représentation des systèmes dynamiques substitutifs non unimodulaires, preprint to appear in Ergodic Theory and Dynamical Systems.

[11] _ Représentation géométrique, combinatoire et arithmétique des substitutions de type Pisot, Ph.D. Thesis, Université de la Méditerranée, 2000 . 